\title{
Modification of intubation techniques during ERCP in patients with Zenker's
}

\section{diverticulum}

\author{
Jamry Andrzej
}

Surgical Department, District Hospital, Radomska, 70 27-200 Starachowice, Poland

\section{CASE STUDY}

Please cite this paper as: Andrzej J. Modification of intubation techniques during ERCP in patients with Zenker's diverticulum. AMJ 2018;11(5):286-288.

https://doi.org/10.21767/AMJ.2017.3283

\section{Corresponding Author:}

Jamry Andrzej

Surgical Department, District Hospital, Radomska, 70 27-200

Starachowice, Poland

Email: jamry@tlen.pl

\section{ABSTRACT}

\section{Performing}

endoscopic

retrograde

cholangiopancreatography (ERCP) in patients with Zenker's diverticulum can be technically difficult and is associated with the risk of oesophageal perforation with subsequent mediastinitis. Therefore, resistance is felt during oesophageal intubation with a duodenoscope, the operator should use a gastroscope to visualize any obstruction. If the existence of an oesophageal pouch is recognised, intubation can be safely performed using a guide-wire over tube technique. However, in specific situations (e.g., narrow oesophageal orifices in patients with low body mass index (BMI)) despite use of guide-wire technique, intubation can still be difficult. Therefore we present a modified guide-wire technique involving rotation of the duodenoscope under fluoroscopy, which opens the entrance to the oesophagus and enables safe intubation.

\section{Key Words}

ERCP, Zenker's diverticulum, oesophageal intubation

\section{Implications for Practice:}

\section{What is known about this subject?}

During endoscopic retrograde cholangiopancreatography
(ERCP) oesophageal intubation is a "blind manoeuvre"; therefore, co-existence of Zenker's diverticulum (ZD) creates a high risk of perforation.

\section{What new information is offered in this case study?}

In specific situations (e.g., compressed oesophageal orifices in patients with low body mass index (BMI) despite use of guide-wire technique, intubation can still be difficult.

3. What are the implications for research, policy, or practice?

In selected cases, complementing the guide-wire technique with proper duodenoscope rotation under fluoroscopy can enable safe oesophageal intubation.

\section{Background}

During endoscopic retrograde cholangiopancreatography (ERCP) oesophageal intubation is a "blind manoeuvre"; therefore, co-existence of Zenker's diverticulum (ZD) creates a high risk of perforation. In most cases, this risk can be minimized by passing the endotracheal tube over a catheter or guide-wire. ${ }^{1-4}$ In cases where the entry to the diverticulum is significantly wider than the entrance to the oesophagus, intubation is a challenge despite the use of this guide-wire technique. In addition, in patients with low BMI use of standard overtube may be impossible. In this situation, complementing the guide-wire technique with proper duodenoscope rotation under fluoroscopy can enable safe oesophageal intubation.

\section{Case details}

A 76-year-old male with a history of intermittent dysphagia was admitted to the surgical department for endoscopic treatment of jaundice with suspected choledocholithiasis. I think it is important to include the patient's BMI or his height and weight. ERCP was initiated using a duodenoscope (TJF 140 Olympus), but intubation was impossible because resistance was encountered just below the upper oesophageal sphincter. The scope was exchanged for a gastroscope (GIF Q 180 Olympus). The presence of Zenker's diverticulum with a wide outlet and narrow C- 
shaped oesophageal orifice was identified (Figure 1); therefore, the guide-wire catheter technique was used. After the guide-wire (Wilson-Coock) was introduced to the oesophagus, the gastroscope was removed, and the proximal part of the guide-wire was introduced in a retrograde manner into the duodenoscope, preloaded with a canniulotome (301 -Q Olympus). Notwithstanding, because of the lateral position of the duodenoscope's working channel outlet, intubation was still difficult. The patient's low BMI made use of the overtube technique impossible, because even the smallest size overtube was too large. Therefore, when the duodenoscope with guidewire reached the upper sphincter, it was rotated around its long axis to a position where the outlet working channel was opposite the diverticulum. In this position, the guidewire/caniulotome complex opened the entrance to the oesophagus and allowed intubation. The ERCP procedure was subsequently carried out without complication.

\section{Discussion}

Zenker's diverticulum (ZD) is a relatively uncommon disorder, with an incidence of 0.01-0.11 per cent, that occurs in people in their seventh and eighth decade of life. ${ }^{5}$ Because duodenoscopy only allows observation of the lateral field of view, ERCP performed in patients with ZD presents a particularly high risk of oesophageal perforation. Therefore, an endoscopist who encounters resistance during intubation is obliged to reposition the scope to achieve a straight on view. If $Z D$ is present, problems with intubation may be overcome by using the guidewire/catheter endoscopic intubation technique. ${ }^{1,2}$ However, if the diverticulum orifice is much larger than the oesophageal orifice, the duodenoscope may not be introduced easily, even with the use of this technique. Tsang and Buto, in 1992 described a hybrid technique in which a guide-wire was introduced first, followed by the use of an overtube, which allowed safe, "blind" intubation. ${ }^{4}$ However, for patients with low BMI, such as the patient described in this report, the classic overtube is too wide; therefore, we propose the technical modification described. The key to this modification is rotation of duodenoscope to a position in which the working channel with the catheter is located opposite the diverticulum. This position opens the oesophageal lumen and allows for safer intubation. A similar manoeuvre is used by some endoscopists performing ERCP in cases where passage of the duodenoscope to the second part of the duodenum is difficult. However, to our knowledge, there are currently no publications describing the use of this technique in the presence of ZD.

\section{Conclusion}

Proper duodenoscopic rotation under fluoroscopic control, together with guide-wire technique, enables safe oesophageal intubation in the presence of diverticulum.

\section{References}

1. Tsang TK, Buto SK. Catheter guided endoscopic intubation: a new technique for intubating a difficult esophagus. Gastrointest Endoscopy. 1992;38:49-51.

2. Malik A, Chitnavis V, Epstein A. Use of a hydrophilic wire for esophageal intubation in Zenker's diverticulum. Gastrointest Endoscopy. 1994;40:523-524.

3. Kadish S, Faigel D, Long W. Safe duodenoscopic intubation in a patient with a large Zenker's diverticulum. Gastrointest Endoscopy. 1996;1:101-102.

4. Dickey W, Porter KG. Duodenoscope intubation of the esophagus in the presence of pharyngeal pouch made possible by an overtube. Endoscopy. 1995;27:272-273.

5. Lerut $T$, Coosemans $W$, Decaluwe $H$, et al. Zenker's diverticulum. Multimed Man Cardiothorac Surg. 2009;224:2881. doi:10.1510/mmcts.2007.002881

\section{PEER REVIEW}

Not commissioned. Externally peer reviewed.

\section{CONFLICTS OF INTEREST}

The authors declare that they have no competing interests.

\section{FUNDING}

None

\section{PATIENT CONSENT}

The authors, Andrzej J, declare that:

1. They have obtained written, informed consent for the publication of the details relating to the patient(s) in this report.

2. All possible steps have been taken to safeguard the identity of the patient(s).

3. This submission is compliant with the requirements of local research ethics committees. 
Figure 1: Endoscopic image of the compressed entry into the oesophagus

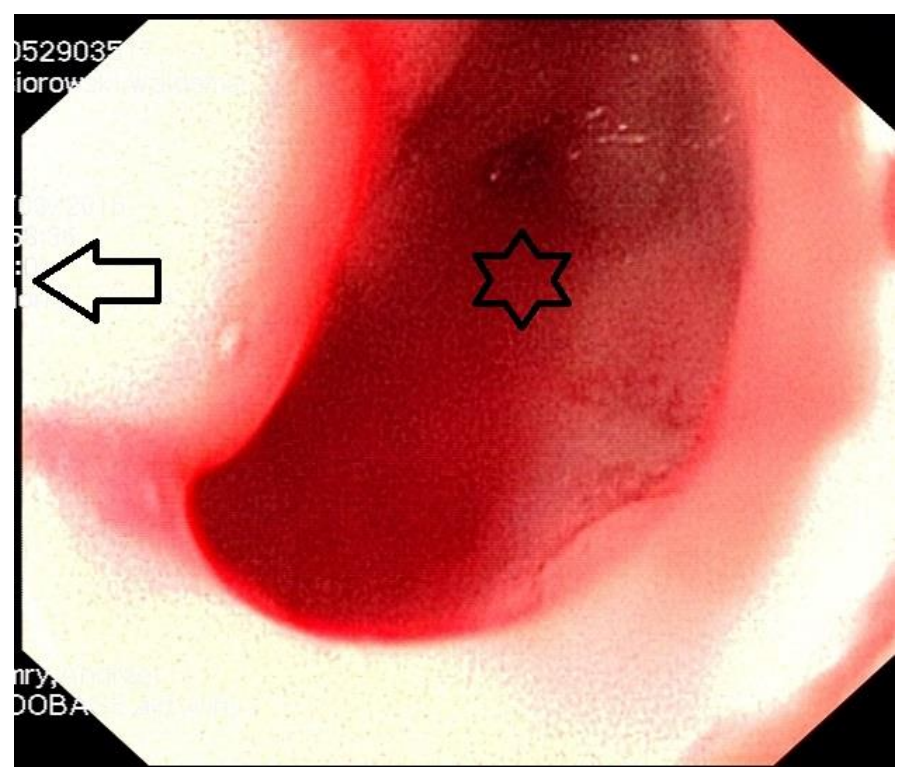

